\title{
URINARY STONE DISEASE
}

\author{
Chris Dawson, Hugh Whitfield
}

\section{Investigation}

\section{Presenting features of urinary stone disease}

- Renal pain is usually felt in the loin, sometimes spreading to the umbilicus and testis. Pain from irritation of the intercostal nerves is not colicky and may be provoked by pressure on a trigger spot

- Obstruction of the mid-ureter may mimic appendicitis on the right and diverticulitis on the left

- Obstruction of the lower ureter may lead to bladder irritability or pain in the scrotum, penile tip, or labia majora

- A recurrent painful desire to micturate, with only a little urine passed each time (strangury)
Most patients with urinary stones have microscopic haematuria detected by routine urine analysis. An absence of red cells in the urine suggests an alternative diagnosis.

\section{Differential diagnosis of renal}

\section{colic}

- Acute appendicitis

- Diverticulitis

- Salpingitis

- Ruptured aortic aneurysm

- Pyelonephritis
An $x$ ray film of the kidneys, ureters, and bladder shows the position and size of the kidneys as well as the presence of any calculi, which usually obstruct at the pelviureteric junction, the point where the ureter crosses the iliac arteries at the pelvic brim, or the vesicoureteric junction. All patients suspected of having a urinary stone should then have intravenous urography, although renal ultrasonography is acceptable in patients who are either allergic to contrast medium or pregnant. Obstruction may be detected by the delayed appearance or persistence of the nephrogram phase on the intravenous urogram or by the finding of caliceal dilatation in an ultrasound scan.

\section{Investigations}

- Urine analysis

- $x$ Ray examination of the kidneys, ureters, and bladder

- Intravenous urography

- Renography

- Metabolic screening

\section{Investigations in high risk patients}

- Serum concentrations of calcium, uric acid, and phosphate

- 24 Hour urine $\mathrm{pH}$ and concentrations of oxalate, phosphate, uric acid, and calcium

- Spot urine test for cystine
Renography after injection of technetium-99m mercapto acetyltriglycine is not routinely used and is more useful when the urogram suggests obstruction, particularly if the stone does not pass quickly.

About two thirds of men presenting with a stone will have recurrent stones. The likelihood of detecting a metabolic abnormality is similar in patients with single and recurrent stones. A compromise for metabolic screening must be reached between the risks of further stone formation on the one hand and the cost of full evaluation on the other. Full metabolic screening should therefore be reserved for high risk groups (such as those with a family history of stones or with recurrent stones). 


\section{Conservative management of stone disease}

Diameter of stone $(\mathrm{mm})$

$\begin{array}{lr}<4 & 90 \\ 4-6 & 50 \\ >6 & 10\end{array}$

Patients should be given analgesics and antiemetics as needed. Nonsteroidal anti-inflammatory drugs provide effective analgesia in most cases, although opiates may be required. Intravenous fluids should be given if a patient cannot tolerate oral fluids, but there is no therapeutic benefit from a regimen of forced diuresis. The increased diuresis that results causes a decrease in ureteric peristalsis, which may lessen the chance of spontaneous passage of the stone. The size of the stone is an important determinant of whether conservative management is likely to succeed-the smaller the stone, the more likely that it will pass spontaneously.

\section{Surgical options}

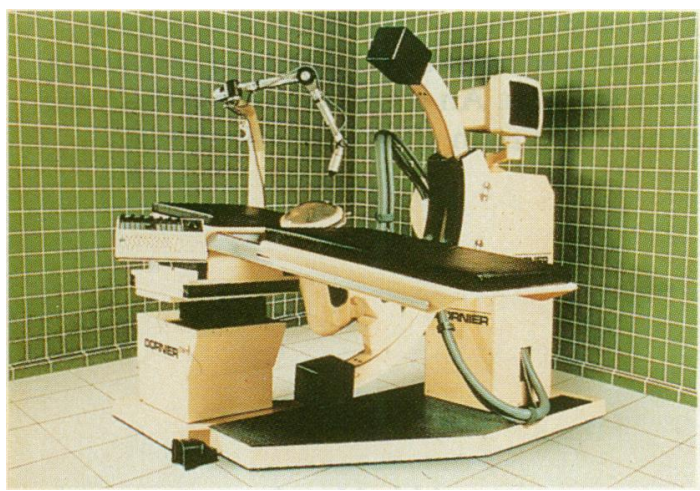

A lithotripter.

\section{Extracorporeal shock wave lithotripsy}

Extracorporeal shock wave lithotripsy has evolved considerably since first described in 1982 and is now the treatment of choice for most renal and ureteric calculi. Most urology departments perform shock wave lithotripsy on a day case or outpatient basis, and non-steroidal anti-inflammatory drugs usually provide sufficient pain relief. Antibiotics should also be given as bacteria are found in more than $30 \%$ of stones. If a patient has been given opiates for analgesia then a four hour recovery period is mandatory, but otherwise he or she can be allowed home immediately after treatment. A written instruction sheet with details of common symptoms is useful, and patients should be told to return to the department or phone for advice if severe pain or signs of infection develop.

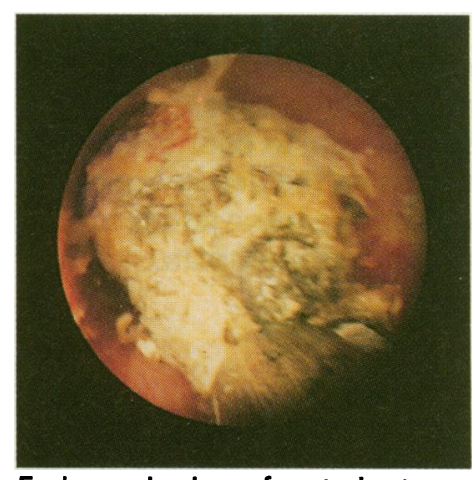

Endoscopic view of ureteric stone. The red light is a focusing beam for a pulsed dye laser, used to disintegrate the stone. $x$ Ray film of dilator passed over guide wire to create percutaneous track into kidney.

\section{Ureteroscopy}

Ureteroscopy has been revolutionised by the development of smaller telescopes, and the procedure is usually carried out as a day case under general anaesthesia. The stone is either removed by capturing it in a wire basket and gently extracting it or fragmented with an ultrasonic probe, laser energy, or a newer, pneumatic instrument known as a lithoclast. An endoscope cannot always be passed up to the stone, however, and the higher the stone, the greater the risk of ureteric damage.

\section{Percutaneous nephrolithotomy}

For percutaneous nephrolithotomy the patient is anaesthetised, and a radiologist or urologist inserts a needle into a lower pole calix of the affected kidney under ultrasound or fluoroscopic control. A guide wire is then passed down the needle, which is exchanged for a series of dilators used to dilate a track through which a nephroscope can be inserted into the renal pelvis. The stone is fragmented under direct vision and the fragments removed. Occasionally extracorporeal shock wave lithotripsy may be needed as a secondary procedure to fragment stones not cleared by percutaneous nephrolithotomy. Stones in the upper third of the ureter may also be best treated with an antegrade approach. 


\section{Indications for open surgery}

- For a stone that cannot be removed by other means

- In a morbidly obese patient (other procedures are technically impossible)

- In a patient whose poor health precludes other (lengthier) procedures

- For large, complex staghorn calculi

Open surgery is now rare

\section{Choice of surgery}

Of the surgical options for urinary stone disease, extracorporeal shock wave lithotripsy is the treatment of choice for most renal stones less than $2 \mathrm{~cm}$ in diameter when no evidence of obstruction exists. For stones larger than $2 \mathrm{~cm}$ or if the caliceal neck is obstructed then percutaneous nephrolithotomy is preferred. Treating renal stones larger than $2 \mathrm{~cm}$ with extracorporeal shock wave lithotripsy is not worth while unless the patient is unfit to undergo another procedure as the results are in general poor, and the risk of ureteric obstruction from stone fragments increases.

\section{Key references}

Drach GW. Urinary lithiasis: etiology, diagnosis, and medical management. In: Walsh PC, Retik AB, Stamey TA, Vaughan ED, eds. Campbell's urology. Philadelphia: W B Saunders, 1992

Preminger GM. Renal calculi: pathogenesis, diagnosis and medical therapy. Semin Nephrol 1992; 12: 200-16
Stones in the upper ureter may be treated with in situ lithotripsy, provided that no evidence of sepsis exists in an obstructed upper tract. If sepsis is present than a percutaneous nephrostomy should be performed urgently to relieve the obstruction and drain the kidney. Retrograde manipulation of the stone into the renal pelvis with a ureteric stent has been largely abandoned as the results of in situ lithotripsy are equivalent, and the morbidity associated with a ureteric stent is thus avoided. Lithotripsy of the mid-ureter is difficult because stones may not be easy to see in this area. Open surgery may be needed because stones located here may be too low for antegrade percutaneous removal but too high for easy ureteroscopic removal. Stones in the lower ureter or at the vesicoureteric junction may be treated with lithotropsy or with ureteroscopy with similar results. Stones in the bladder may be treated initially with lithotripsy. Most bladder stones result from bladder outflow obstruction (for example, due to benign prostatic hyperplasia or a urethral stricture), and this condition should also be treated to prevent a recurrence.
The ABC of Urology is edited by Chris Dawson, a senior registrar in urology at the Edith Cavell Hospital, Peterborough, and Hugh Whitfield, a consultant urologist at the Central Middlesex Hospital and the Institute of Urology and Nephrology, London.

\title{
Lesson of the Week
}

\section{Recurrent pelvic endometriosis and bilateral ureteric obstruction associated with hormone replacement therapy}

\author{
RJ Brough, K O'Flynn
}

Endometriosis after the menopause may be reactivated by hormone replacement therapy
Department of Urology, Manchester Royal Infirmary, Manchester M13 9WL

RJ Brough, research registrar K O'Flynn, senior registrar

Correspondence to: Mr Brough.

$B M 71996 ; 312: 1221-2$
Between $2 \cdot 5 \%$ and $15 \%$ of women of reproductive age have endometriosis, ${ }^{1}$ yet the ureter is only rarely affected. ${ }^{2}$ We report the case of a patient presenting with bilateral ureteric obstruction secondary to recurrent endometriosis. She had previously undergone a total hysterectomy and bilateral salpingooophrectomy for endometriosis and had been taking unopposed oestrogen for postmenopausal symptoms in the five years after surgery.

\section{Case report}

A 47 year old health care worker was suddenly admitted to hospital; she complained of three months of increasing bilateral loin pain, change in bowel habit, and general malaise. Six years earlier she had had a hysterectomy and bilateral salpingo-oophrectomy for endometriosis. Surgery showed that she had extensive disease in the pelvis with adhesions obliterating the pouch of Douglas. She was given a six month course of danazol because of the likelihood of residual disease. Histological examination of the ovaries and uterus showed extensive endometriosis affecting the peri- toneum and both ovaries, with haemorrhagic adhesion throughout the specimen. Hormone replacement therapy in the form of a combined oestrogen and progestogen pill was offered after the course of danazol, but the patient declined.

Two years after surgery she complained of hot flushes, irritability, and vaginal soreness. Her general practitioner diagnosed postmenopausal symptoms, and she was started on a course of regular oral oestrogen (ethinyloestradiol, $10 \mu \mathrm{g}$ a day). Shortly afterwards she began to have intermittent right loin pain and was referred to the general surgeons, who diagnosed early diverticular disease on colonoscopy and took no further action. She continued taking hormone replacement therapy up to the time of her acute admission.

At that time pelvic examination showed a lower abdominal mass, anterior to the rectum. Serum urea and electrolyte concentrations were normal. An intravenous urogram and renogram showed the right kidney to be completely obstructed and contributing only $11 \%$ to overall renal function. The left kidney was partially obstructed and contributing $89 \%$ of all renal 